"This is a post-peer-review, pre-copyedit version of the article Berberian B., Ohneiser O., De Crescenzio F., Babiloni F., Di Flumeri G., Hasselberg A. (2017) MINIMA Project: Detecting and Mitigating the Negative Impact of Automation published in: Harris D. (eds) Engineering Psychology and Cognitive Ergonomics: Performance, Emotion and Situation Awareness. EPCE 2017. Lecture Notes in Computer Science, vol 10275. Springer, Cham.

The final authenticated version is available online at: https://doi.org/10.1007/978-3-319-58472-0_8".

Link at the published version: https://link.springer.com/chapter/10.1007\%2F978-3-319$58472-0 \quad 8$ 


\title{
MINIMA project: detecting and mitigating the negative impact of automation
}

\author{
Bruno Berberian $^{1 *}$, Oliver Ohneiser ${ }^{2}$, Francesca De Crescenzio ${ }^{3}$, Fabio Babiloni ${ }^{4}$, \\ Gianluca Di Flumeri ${ }^{4}$, Andreas Hasselberg ${ }^{2}$ \\ ${ }^{1}$ French Aerospace Lab (ONERA), Salon, France \\ ${ }^{2}$ German Aerospace Center (DLR), Braunschweig, Germany \\ ${ }^{3}$ University of Bologna, Forlì, Italy \\ ${ }^{4}$ BrainSigns srl, Roma, Italy \\ Corresponding author: bruno.berberian@onera.fr
}

\begin{abstract}
In this paper, we present the preliminary steps conducted in the framework of the research project Mitigating Negative Impacts of Monitoring high levels of Automation (MINIMA). The main objectives of MINIMA are i) to develop vigilance and attention neuro-physiological indexes, and ii) to implement them in a system that can adapt its behavior and guide the operator's attention. The goal is to mitigate negative impacts of the foreseen increasing automation in future Air Traffic Control (ATC) scenarios on Air Traffic Controller (ATCo) performance. The first step of research activities consists of better comprehension of Out-Of-The-Loop (OOTL) phenomena and of current methods to measure and compensate such effects. Based on this State of the Art, we propose the MINIMA concept, i.e. a dynamic adaptation of the task environment which is foreseen as a major requirement to keep the human 'in the loop', perfectly aware of the traffic situation. In the second part of this paper, we give details about the highly automated Terminal Manoeuvring Area selected as case study. Additionally, we describe the adaptation mechanisms that are planned to be implemented into this task environment and analyzed in the MINIMA project. Finally, the document provides information about the technical implementation of the vigilance and attention measurement that will be used to trigger adaptation of the task environment.
\end{abstract}

Keywords: Air Traffic Controller; Terminal Manoeuvring Area; Automation; Vigilance; Attention; Adaptive Task and Support Activation; Attention Guidance; Electroencephalography

\section{Introduction}

Over the past few years the global air traffic growth has exhibited a fairly stable positive trend, even through economic immobility, financial crisis and increased security concerns. According to a prevailing opinion, this trend is unlikely to change in the

adfa, p. 1, 2011.

(C) Springer-Verlag Berlin Heidelberg 2011 
future, although a number of contextual factors, such as political climate, economy, environmental issues, safety issues and security issues may affect its actual rate. Further, according to the 'Free Flight' and the '4D Trajectory Management' concepts, different types of aircraft, such as manned, unmanned, and autonomous aircraft, as well as all kinds of rotorcrafts, will operate simultaneously in a 'structure-less' and 'time based' environment [1-2] allowing for much more direct and continuous trajectories to be used. Also, brand new airspace designs, possibly dynamic, may be required.

Within this picture, traffic flow patterns will become more complex, making conflicts and situations harder to identify for a human operator, putting immense pressure on the air traffic control system. Several solutions have been proposed for modernizing air traffic control and meet the demands for enhanced capacity, efficiency, and safety. As envisaged by both SESAR JU and HALA! Research Network, higher levels of automation will help ATCos to deal with increasingly complex airspace scenarios, enabling them to manage complex situations in a safe and efficient way.

In the present case, the changes in the ATC environment will also cause a shift of Air Traffic Controllers' (ATCo) tasks from active managing of aircraft to monitoring [3]. In the future, ATCos' actions will only be necessary if an aircraft deviates from its scheduled plan. However, ATCos being less actively involved in the ATC task may be affected by the Out-Of-The-Loop phenomena including performance degradation during their work. Such a new ATCo may show a "diminished ability both to detect system errors, and subsequently to perform manual tasks in facing automation failures, compared with operators who normally perform the same tasks manually" [4].

MINIMA will address these performance issues. Its aim is to identify thresholds in future ATM scenarios identifying out-of-the-loop behavior and to find solutions to minimize the negative impact of monitoring high levels of automation on the human operator's performance. In the following parts, we will first describe the performance consequences of the OOTL phenomenon and discussed how to explain such degradation. Then, we will present solutions described in the literature to mitigate OOTL phenomenon. Finally, we will propose a technical solution to detect and compensate this OOTL phenomenon.

\section{OOTL Characterization}

Increasing the automation of ATM will result in new roles for ATCo. They will mainly monitor highly automated system and intervene seldom. Such change (from manual to supervisory control) is far from trivial. The key difference between passive information processing and direct action on the process is that the former involves functions similar to those maintained during process monitoring (e.g., scanning information sources); whereas, the latter involves manual control functions including process planning, decision making, selecting responses and implementing strategies. The problems due to automation are related to these new roles that are created for operators when their tasks are changed from manual to supervisory control. 
Indeed, empirical data suggest that human operator is not always very efficient in supervisory task [5-6]. Particularly, several incidents highlight that when the automatic equipment fails, supervisors seem dramatically helpless for diagnosing the situation and determining the appropriate solution because they are not aware of the system state prior to the failure. Numerous experimental results confirm such difficulties. For example, Endsley and Kiris [5] provided evidence that performance during failure mode following a fully automated period were significantly degraded, as compared to a failure mode following a fully manual control. Merat and Jamson (2009) reported similar conclusions. In a driving simulation task, they demonstrated that drivers' responses to critical events were slower in the automatic driving condition than in the manual condition.

This so called Out-Of-The-Loop (OOTL) performance problem represents a key challenge for both systems designers and human factor society. However, after decades of research, this phenomenon remains difficult to grasp and treat. In the following section, we aim to bring a better understanding of this crucial phenomenon.

\subsection{Becoming out of the loop}

In the current research, we consider this phenomenon as it occurs in the context of human machine interaction. The human involvement and role in control of automatic systems depends on the level of automation. In manual control, the operator acts on the object, hence performing control on a lower level of aggregation. When a high level of automation is used, all of the control levels mentioned above are active and the operator acts as a supervisor on a high level where system and sub-system functionality is monitored rather than individual objects. The automation took care of the lower level actions and the human operators simply watched over the system, presumably ever-alert for deviations and problems. In other words, operators are relegated to passive information processor: they are "out of the loop" [8-10].

In other words, the OOTL phenomenon corresponds to a lack of control loop involvement of the human operator. Automation technology has created an increasing distance between human operator and loop of control, making him disconnected from the automation system. Such a removal leads to a decreased ability of the human operator to intervene in system control loops and assume manual control when needed in overseeing automated systems (see following sections). Because automation is not powerful enough to handle all abnormalities, this difficulty in takeover is a central problem in automation design.

\subsection{OOTL performance problem and decrease in Situation Awareness}

The origins of these takeover difficulties have been largely debated. In the current accepted picture, the degradation of the Situation Awareness (SA) appears as a key component to understand the OOTL performance problem. Situation awareness is defined as "the perception of elements in the environment within a volume of time and space, the comprehension of their meaning, and the projection of their status in the near future" [11]. Situation awareness encompasses three processes: the percep- 
tion of what is happening (Level 1), the understanding of what has been perceived (Level 2) and the use of what is understood to think ahead (Level 3).

Nowadays, it is clear that a loss of situation awareness underlies a great deal of the out-of-the-loop performance problem and that OOTL phenomenon is characterized by both a failure to detect and to understand the problem and by difficulties to find appropriate solutions.

\section{Failure to detect (Level 1).}

Numerous incidents have been attributed to a lack of operator awareness of automation failures and a decrease in detection of critical system state changes when involving in automation supervision (for a review see [5]). The near crash of Air China's Boeing 747 in 1989 illustrates this difficulty. In this case, the aircraft experienced a gradual engine failure that the human pilot was not aware of because of autopilot compensation (through rudder control) up until the point of failure of the autopilot, itself. Subsequently, the jet stalled and plummeted thousands of feet, being recovered within a few seconds of the ocean surface [12]. Numerous empirical evidences confirm that ATCos may be poor in detecting aircraft-to-aircraft conflicts when they are not actively controlling the airspace but nevertheless have to monitor for occasional anomalies. As illustration, Endsley and Rodgers [13] found that ATCos showed poor performance in detecting conflicts in recorded traffic when they were passively monitoring the traffic. Galster et al. [14] found that passive monitoring with airborne control of aircraft separation, which would be the case under mature Free Flight, led to a marked decrease in conflict detection performance by ATCos under high traffic load.

Failure to understand (Level 2 and Level 3).

In addition to delays in detecting that a problem has occurred necessitating intervention, operators may meet difficulties to develop sufficient understanding of the situation and to overcome the problem. For example, Wickens and Kessel [15] demonstrate longer system recovery times and poor response accuracies for operators who had been removed from control loops in advance of critical events requiring intervention. This delay may prohibit operators from carrying out the very tasks they are required to perform or diminish the effectiveness of actions taken.

Further, during failure modes, operators who have been removed from system control may not know what corrective actions need to be taken to stabilize the system and bring it into control. Several examples of incidents and accidents resulting from these system misunderstandings have been reported [16-18]. "Automation surprises" are a direct instantiation of these difficulties in automation understanding and take-over situations [19] and correspond to situation where the operator is surprised by the behavior of the automation. These "automation surprises" are particularly well documented [20-22] and have been listed as one of the major cause of incidents.

\subsection{Origins of The Out-Of-The-Loop performance problem.}

Changes in attention/vigilance mechanisms appear as a first concern to explain the OOTL performance problem and the SA deterioration. And for good reasons since 
research on vigilance has shown that humans are poorly suited for monitoring role [23]. Pilots' reports of incidents have notably highlighted difficulties in monitoring automated systems. For example, Mosier and collaborators [24] examined NASA's Aviation Safety Reporting System database and found that $77 \%$ of the incidents in which over-reliance on automation was suspected involved a probable vigilance failure. Similarly, Gerbert and Kemmler [25] studied German aviators' anonymous responses to questionnaires about automation-related incidents and reported failures of vigilance as the largest contributor to human error. Two main sources could explain such decrease in vigilance: the inability to maintain a high level of vigilance in time and the complacency effect.

\section{Difficulties to maintain high level of vigilance.}

One unintended consequence of automation for human operators is boredom. Indeed, highly automated environments require maintaining high levels of vigilance during a long period of time. In many phases of operation, operators are reduced to monitoring activities, waiting for the unlikely system anomaly. Resulting boredom increases the likelihood of operator distraction, which ultimately can affect system performance if operators miss or respond late to critical events.

Interestingly, several studies show that sustained attention over hours cannot be achieved [23][26]. Research on vigilance suggests that time on task decreases significantly the discrimination of infrequent and unpredictable signals from a noisy background of nonsignals [27-29]. Moreover, there is some consensus for the existence of a decrease of human operator vigilance in case of interaction with highly automated system [30-32]. Both change in vigilance level and deterioration of the attentional mechanisms could cause degradation of the monitoring process involved in supervisory task and decrease performance in failure detection and system understanding.

\section{Over-trust / complacency.}

Together with this difficulty to maintain high level of vigilance in time, decrease in vigilance could also result from an overreliance on automation, the so-called complacency phenomenon [33]. Complacency defines the cognitive orientation toward high reliability automation, particularly prior to the first time it has failed in the user's experience [34]. Overreliance or complacency is created as operators form beliefs of the technical system as being more competent than it actually is. This overreliance on automation represents an important aspect of misuse that can result from several forms of human error, including decision biases and failure of monitoring [35-37]. A typical illustration is the case of the crash of Northwest Airlines at Detroit Airport in 1987. The McDonnell Douglas MD-80 crashed due to improper configuration of the flaps and slats of the aircraft. All persons were killed because an automated take-off configuration warning system, which the crew relied on, failed to function. They did not realize the aircraft was improperly configured for take-off and failed to check manually [38]. In this case like in others, when computer control facilities failed, operators, out of the direct control loop, were unaware of the state of the system and encounter difficulties to compensate for the failure mode before an accident occurred. 
The previous section illustrates how the lack of operator involvement in automated systems control and the vigilance decrements induced will contribute to the loss of operator situation awareness. The following will present the different solutions currently proposed to mitigate this OOTL performance problem.

\section{Current solutions for OOTL mitigation}

Solutions for solving the OOTL-Problem are diverse. Following the perspective of an "Interaction Problem", a solution can either target at the system or target at human operators to make them less prone to OOTL-Problems.

\subsection{Human operator "adaptation"}

A first solution for OOTL problems targeting human operators is acting on their training. Human operator can be explicitly trained for situations in which OOTL problems can occur. For example, in a laboratory experiment using a process control simulation, Bahner and colleagues [39] showed that a preventive training in which participants were exposed to rare automation failures could significantly reduce complacency. Careful selection of the operators is presented as another solution targeting the human relates. Today, ATCos are carefully selected based on the key ability required by the working environments. Regarding the increase in automation, the ability to monitor automated systems and to switching immediately from monitoring to decision making will become an important competence in the selection of future ATCos [40]. If promising, such solution needs time to become effective and empirical evidence needs to be collected regarding its effectiveness.

\subsection{System adaptation}

Since OOTL problems are caused by changing the system and introducing higher levels of automation, it seems likely that it can also be solved by changing the systems. For example, MABA-MABA-like methods (Men Are Better At-Machines Are Better At) rest on the idea that you should exploit the strengths of both humans and machines differently. The basic premise is: give the machines the tasks that they are good at, and the humans the things that they are good at (see for example [41]). However, Dekker and Woods [42] argued that such methods are misleading as automation often has unexpected effects [43]. These include the OOTL problems discussed above. It is now clear that introducing automation does not simply transfer the execution of functions to the machine, but instead create completely new functions and transform human practice. They conclude that automation needs to support cooperation with human operators - in standard and unexpected situations. Also, Rieth and collaborators [44] argued for better design of Human-Machine-Systems. They showed that the visual salience of standard indicators "generally do not draw attention to the information needed to identify emerging problems" and suggested other formats by which better mapping the task-relevance of information to the visual salience of how 
it is displayed. A holist approach is to develop automation in such a way that it can be seen as a partner. Human operator and automation should form a team that works cooperatively together, in a highly adaptive way to achieve its objectives. They have to adapt to each other and to the context in order to guarantee fluent and cooperative task achievement. For example, Klein and colleagues [45] defined ten challenges to improve human machine cooperation (model the others' intentions, be delectable, make their status and intentions obvious and be able to interpret the status and intention of others, be able to engage in goal negotiations and enable a collaborative approach, be able to participate in managing attention, and help controlling the costs of coordinated activity).

\subsection{Adaptive Automation as a solution}

A system that can be considered as cooperative must be able to adapt to the needs and the state of the user in real time. It is able to meet the changing needs of operators often without requiring the human operator to explicitly state his needs or trigger the adaptations. Making a system adaptive enables it to behave like a good human assistant. A technical solution for some of these challenges is the concept of adaptive systems.

The concept of Adaptive Automation (AA) concentrates on the dynamic allocation of function between operators and systems. This means, in that the Level of Automation of such system is not fixed but is adapted during the runtime according to the current needs of the operator. Particularly, the level of automation of such system is not fixed, but it is adapted during the activity according with the current needs of the operator [46]. Consequently, adaptive automation enables the level or modes of automation to be tied more closely to operator needs at any given moment [47] without requiring the human operator to explicitly state his/her needs or trigger the adaptations. Several evidences have proved the AA can improve operator's performance and moderate workload in complex environment [48-49]. Besides the dynamic allocation of functions, other aspects of a system can be adapted during operations like, for example, the modality which is used to provide information, the amount of information that is presented to the operator or the lay-out of the information. The MINIMA project aims to design such cooperative/adaptive system. In the last section, we will describe the solution proposed in MINIMA.

\section{MINIMA Concept}

MINIMA will develop a dynamic adaptation of the task environment which is foreseen as a major requirement to keep the human 'in the loop', perfectly aware of the traffic situation. As a consequence of the developed concept, not all tasks potentially automated will be automated every time. To trigger adaptations of the automation, MINIMA will develop a real-time monitoring system that constantly measures the operators' state regarding the OOTL phenomenon. Because vigilance decrement is considered as one the major index of OOTL phenomenon, we will focus on vigilance 
and attention levels. This is called "Vigilance and Attention Observer" in MINMA. A component called "Adaptive Task and Support Activation" will decide based on the measured vigilance and attention level which adaptions of the task environment should be activated. On Overview of the MINIMA Concept is shown in Fig. 1.

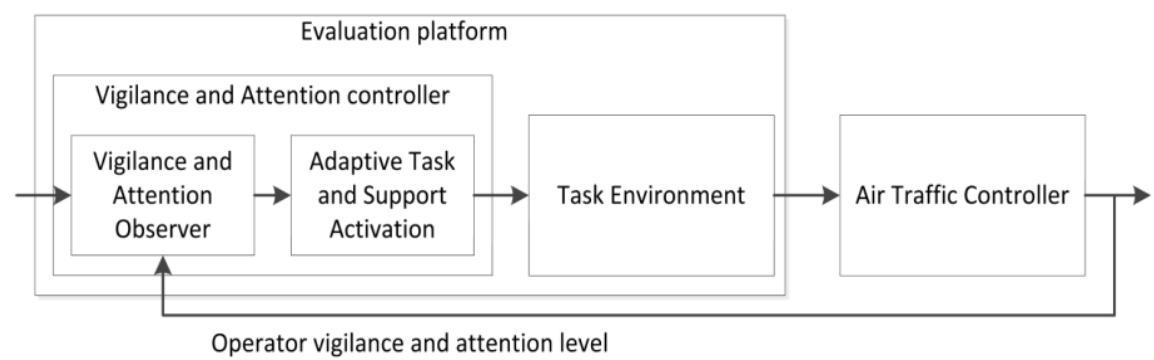

Fig. 1. Evaluation environment for MINIMA and influences of components on others

\subsection{Use case selected in MINIMA}

A highly automated Terminal Manoeuvring Area (TMA) has been selected as use case in MINIMA. This task environment represents an air traffic control task as it is expected for the future: Most of the interaction with the aircraft is automated. A principle assumption of MINIMA is that Air Traffic Controllers (ATCos) are required to intervene in a few situations as error-free automation cannot be guaranteed.

In the MINIMA use case, the arrival management will be highly automated. Onboard Flight Management Systems (FMS) will negotiate with an Arrival Manager (AMAN) on 4D-Trajectories automatically. However, these trajectories are only guaranteed to be conflict-free at a merging point. Conflicts between arrival aircraft at other locations, conflicts between arrival and departures, and deviations form 4DTrajectoreis are still possible - but seldom - and need to be managed by the ATCos.

One controller will be responsible for monitoring arriving and departing traffic. $\mathrm{He} / \mathrm{She}$ only needs to intervene in cases of conflicts or emergencies.

\subsection{Vigilance and Attention Observer}

A critical challenge when designing such adaptive automation is to determine how changes among modes or levels of automation will be triggered. In other words, what should determine and "trigger" allocation of functions between the operator and the automation system. In MINIMA, we propose to use biophysical measure of vigilance and attention to trigger changes among the modes of automation, a module called Vigilance and Attention observer.

The importance of vigilance decrement for understanding human performance in a variety of industrial and military systems is now largely accepted. Several studies have shown that accidents ranging in scale from major to minor are often the result of vigilance failures [50]. Hawley [51], for example, described the role of vigilance and situation awareness in fratricide incidents in the Iraq war involving the highly auto- 
mated Patriot missile system. Moreover, vigilance decrement is also considered as one the major index of OOTL phenomenon as described previously. In MINIMA project, we assume that both change in vigilance level and deterioration of the attentional mechanisms could cause degradation of the monitoring process involved in supervisory task. In this context, the aim of the operator vigilance and attention level observer is to measure both the current vigilance and attention levels of the human operator in view to quantify the OOTL phenomenon.

\section{Biomarkers of vigilance/attention}

Defining a relevant index of alertness and sustained attention level in an operational context could be considered as the first step required for the development of such an inference system. In the method propose in MINIMA, physiological signals that reflect central nervous system activity would serve as a trigger for shifting among modes or levels of automation. Several reasons explain our choice. First, the measures can be obtained continuously with little intrusion [52-53]. Second, these measures have been found to be diagnostic of multiple levels of arousal, attention, and workload. Even if there are still many critical conceptual and technical issues (e.g., making the recording equipment less obtrusive and obtaining reliable signals in noisy environments), numerous works have proved that it is indeed possible to obtain indices of one's brain activity and use that information to drive an adaptive automation system to improve performance and moderate workload in complex environment [48][54].

Our literature review identified several biopsychometrics sensitive to changes in vigilance/sustained attention suggesting them as potential candidates for triggering adaptive automation such as electroencephalographic (EEG), near-infrared spectroscopy (NIRS), transcranial Doppler sonography (TCD), oculometrics, electrocardiogram (ECG) or skin electric potential (GSR). Regarding both advantages and disadvantages of the different techniques available, we decide to use EEG as biomarkers of change in vigilance. Compared to others neuroimaging devices, EEG offers the best compromise between spatial and temporal resolution, practical use and cost. Together with the use of EEG for vigilance monitoring, we propose to use eye tracking techniques to take into account how the available attentional resources is used by ATCo.

\section{EEG and Vigilance.}

Electroencephalography (or EEG) is the recording of electrical activity produced by the firing of neurons within the brain. Several studies have demonstrated the suitability of EEG for real-world monitoring of mental states [55] and for brain-computerinterface (BCI) applications [56-57].

Interestingly, EEG is assumed as one of the most reliable indicators of vigilance [58] and a number of EEG markers have been specifically correlated with vigilance. Particularly, change in power spectral densities (PSD) within the classically defined frequency bands (alpha, beta, theta, delta, and gamma) or ratios between these frequencies bands seem directly linked to change in vigilance state. Nowadays, there is a very large literature concerning the relationship of oscillatory activity and attention/vigilance [59-61] and brain dynamics associated to vigilance are well known. The general evidence is that lower levels of vigilance are related to increases in lower 
frequencies (theta and alpha) in EEG spectrum [62]. Several BCI systems have been designed based on this idea [56-57][63-64]. Interestingly, these studies show that mental (de)activation may be monitored by changing balance between brain activity regions. Beta activity $(12-30 \mathrm{~Hz})$ is predominant when the participant in the study is generally awake and alert, while the activity dropping to Alpha activity $(8-12 \mathrm{~Hz})$ indicates developing drowsiness, and going further down into the theta region (5-8 $\mathrm{Hz}$ ) may even lead to falling asleep.

In conclusion, summarizing the evidences in order to set a proper experimental design, vigilance could be evaluated investigating theta, alpha, and beta activity on the frontal, frontotemporal and parietal sites of the brain, thus the EEG system will be set up accordingly.

Technical implementation of Vigilance Measurement.

The vigilance monitor device proposed will be based on the power spectral density (PSD) distribution. It will encompass four functions (see Fig.2):

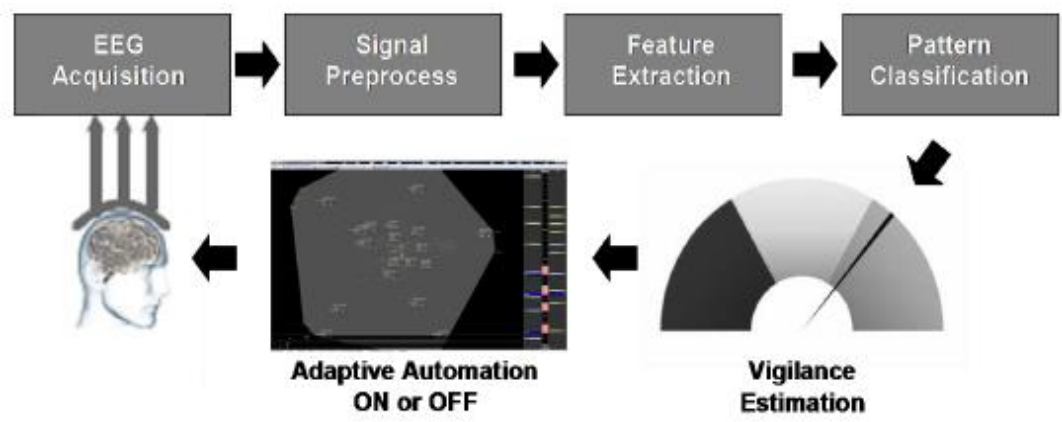

Fig. 2. General Framework of the proposed BCI system for vigilance analysis and estimate. Multi-channels raw EEG signals are first collected as inputs, and then EEG signal must be denoised and removed artefacts. After the preprocess of EEG data, vigilance level would be analyzed by feature extraction and pattern classification algorithms, the results would trigger the Adaptive Automation when the vigilance decrement condition occurs.

- Function 1: EEG acquisition and amplifying,

The EEG device used will be the Galileo BEPlus (EB Neuro Spa, Italy), a system able to record up to 64 EEG channels with a sampling frequency up to $1024 \mathrm{~Hz}$. Galileo BEPlus is a wired EEG system with wet electrodes. The sample frequency will be $256 \mathrm{~Hz}$. EEG caps of different size will allow recording correctly the signal from different subjects independently of their head size. We will first use a high number of electrodes (between 30 and 40) in order to investigate deeply the brain sites involved in the vigilance process. Then, once relevant signal identified and algorithms trained, we will propose to decrease the number of electrodes to 16 electrodes in order to reduce the intrusiveness and improve the wearability of the system proposed. The electrodes will be placed based on the Standard International 10-20 system, mainly on the 
prefrontal, frontal, frontotemporal, and parietal sites. Electrodes in mastoids will be used as reference.

\section{- Function 2: Noise and Artefact elimination}

Removing the polluted signal segments or contaminated channels and reducing the noise influences from EOG, EMG and other channels are all necessary in the EEG signal preprocessing work. In the process of data preprocessing, we will first remove the EEG signals from the damaged channels. Then, the EEG signals will be band-pass filtered (5th order Butterworth filter) to eliminate the noise. Because the brain potential is generally between $1 \mathrm{~Hz}$ and $40 \mathrm{~Hz}$, so the band-pass filter cut-off frequencies are set to $1 \mathrm{~Hz}$ and $40 \mathrm{~Hz}$. At this point, artefacts elimination will be performed. The EEG signal will be segmented into epochs of 2(s), shifted of 0.125(s). The Fpz channel will be used to remove eyes-blink artefacts from the EEG data by using the regression-based algorithm REBLINCA [65]. For other sources of artefacts (i.e., ATCos normally communicate verbally and perform several movements during their operational activity), specific procedures (Threshold criterion, Trend estimation, Sample-to sample difference) available in the EEGLAB toolbox [65] will be applied.

- Function 3: Feature extraction

To make sense of the recorded EEG signal, feature extraction and data dimension reduction are needed. Due to the close relationship between the EEG spectrum and the subject's vigilance state, the rhythm activities, that is, EEG power in the three specified bands, Theta $(4-8 \mathrm{~Hz})$, Alpha $(8-13 \mathrm{~Hz})$, and Beta $(13-30 \mathrm{~Hz})$ and their ratios, are calculated as features. In MINIMA, the EEG Power Spectral Density (PSD) will be estimated by using the Fast Fourier Transform (FFT) in the EEG frequency bands. Such frequency bands will not be defined equally for all the subjects (e.g., alpha equal to the $8 \div 12$ [Hz] band), but the Klimesch approach will be adopted by using the Individual Alpha Frequency, in order to take into account the physiological subjective aspects of brain activity [66].

\section{- Function 4: Pattern classification algorithms}

Various classifiers performing classification task have been proposed. Amongst other, support vector machine (SVM), artificial neural networks (ANN) and autoregressive (AR) are the most commonly used classifiers in the EEG research domain. In the case of MINIMA, a machine learning approach will be thus adopted in order to compute a Vigilance index based on the selected brain features. Particularly, during the calibration scenarios (LowVigilance and HighVigilance), we calibrate the algorithm before the Testing scenarios presentation. In particular, the Power Spectral Density of EEG epochs related to each calibration scenario (LowVigilance and HighVigilance) will be calculated by using only the frequency bands directly correlated to the vigilance state. The EEG frequency bands [frequency resolution of $0.5(\mathrm{~Hz})$ ] of interest will be defined for each ATCo by the estimation of the Individual Alpha Frequency value, as stated previously. At this point, the classification algorithm automatic stop Stepwise Linear Discriminant Analysis (asSWLDA, [67]) will be used to identify the most relevant discriminant features among the two different experimental condi- 
tions related to the lowest and the highest level of vigilance. Once identified, the asSWLDA classifier will assigns to each significant feature specific weights plus a bias. These parameters will be used later on to compute online the vigilance level index of the user during the testing scenarios.

\section{Oculometric measure and attention.}

Often at work, it is asked to the operator to attend more than one thing at the same time. This is possible thanks to the human ability to move the attentional focus, and is essential for every multitasking activity [68]. Of course, measuring only the attentive intensity aspect, i.e. the vigilance in our case, does not pledge that this amount of vigilance is properly oriented toward the tasks composing the work activity: On the contrary, other distracting activities could cause great levels of vigilance but consequently low performance on the work activity itself. For such a reason, it is crucial to monitor also which is the attentional focus of the operator, i.e. where his/her visual attention is addressed. In this context, we propose to use eye tracking device.

Tracking eye movements has the potential to provide a more direct measure of where attention is deployed since the direction of gaze is generally considered to be tightly coupled to the orienting of attention - at least, under normal circumstances. It can provide crucial information about the "attentional path" of the subject, revealing for example if the gaze is correctly directed and if there are particular fixation points [69]. Uncoupling of gaze direction and attention can, of course, occur as Posner's task clearly demonstrates. The value of eye tracking is that in natural scene viewing where the visual environment is complex compared to many simple experimental situations - it should provide a good guide to the locus of attention. In recent years, researchers have capitalized on this possibility, seeking eventually to understand how attention and gaze are deployed to make sense of the visual world.

\section{Technical Implementation of Visual Attention Measurement.}

In MINIMA, we choose to use a remoted system (Tobii EyeX) with $60 \mathrm{~Hz}$ sampling rate. This system can deliver information in real time (i.e., gaze direction). In contrast to EEG signal, the interpretation of eye tracking data is something relatively easy. Even if many different methods of exploring eye data exist, the eye tracking data do not require any particular skill in terms of analysis, since the software itself provides results in terms of gaze movements, fixations and so on. For MINIMA, we propose to use eye tracking to identify in real time where, when and what people look at and what they fail to see.

Basically, we will use fixation and saccades as measures of visual attention and interest. Based on fixation position (where?) and timing information (when?), we could compute different index, like the time and the number of fixation spent on a specific area of interest (AOI) or the Time to First Fixation (or TTFF), that is the amount of time it takes a respondent to look at a specific AOI from stimulus onset. These different metrics will inform us where and when the ATCos look at and how different events in the simulation will catch attention of the ATCo.

Using these measures of the controller attention area, we aim to help the controller in keeping his attention at the relevant display areas. In case of critical event and 
whatever the current level of vigilance, three different situations could be envisaged: (1) ATCo is focused on the relevant part of the radar display regarding the current situation, (2) ATCo is focused on a non-relevant area of the radar display, (3) ATCo is exploring the environment without specific area of interest. Oculometric measure could help us to detect problematic situation as cases 2 or 3 and help ATCo to focus on the relevant information. To make it possible, we need (1) to evaluate which ATC event on the radar display is the most relevant for the controllers, (2) to detect which radar areas the controller actually focuses his attention on, e.g. via eye-tracking, and (3) to guide the controllers' attention to the relevant radar display area if his attention is somewhere else. How to guide vigilance and attention is precisely the purpose of the following section.

\subsection{Attention vigilance guidance module}

In MINIMA, we propose to design a module, called the adaptive task and support activation, able to modify both the level of automation and the feedback sent by the automation technology in order to maintain the ATCos in the loop of control and improve their performance in monitoring task.

In order to identify relevant vigilance and attention guidance tools, a workshop concerning the future ATC was first conducted with four ATCos ( 2 female, 2 male, average age of 39.3 years, average work experience as an ATCo of 20.5 years). The following sections briefly depict some of the ideas for vigilance and attention guidance within MINIMA, derived from the workshop results.

- Attention guidance to separation conflicts (Short term conflict prediction):

Whenever a situation arises that is classified as potential loss of separation or might become a future loss of separation if the ATCo does not interact, the system highlights the affected aircraft. In order to do so, the system calculates the distances between all aircraft based on the current position and the predicted trajectories and detects all separation conflicts between these trajectories. A separation conflict is detected and highlighted if the distance between two aircraft is predicted to be below 3 NM horizontally and below $1000 \mathrm{ft}$ vertically.

- Attention guidance to aircraft that cannot meet agreed target times:

This adaptation is also based on prediction. It is checked if the position of aircraft on their route is according to the last agreed trajectory. If there is a difference, there is the risk that this aircraft might be too late or too early and that it will cause a conflict with another aircraft. If deviations above a threshold of $0.5 \mathrm{NM}$ are detected, the aircraft is highlighted. For example, the difference is shown in the aircraft label.

- Attention guidance with eye tracker:

The system monitors the eye movement of the operator and determines, based on a normative model, which areas are monitored insufficiently or not at all (monitoring loss). Additional information is provided to the ATCo to update his/her situation awareness in this specific area. 
- Centerline Separation Range:

The Centerline Separation Range (CSR) [70] is a visual hypothetical aircraft final visualization (see Fig. 3) similar to HungaroControl's tool MergeStrip [71]. The calculated remaining flight distances of all approaching aircraft relative to other aircraft are displayed as distance-to-go (DTG) in nautical miles on one single arrival flow line for each runway (white numbers). When summing up all DTGs of previous aircraft in the sequence, the DTG of the current aircraft results (e.g. sum up $1.4+7.54+8.01$ to get the DTG of DLH500). The angle brackets indicate the trend of increasing or decreasing relative separation between two sequenced aircraft since last radar update. Aircraft are colored depending on their weight category (e.g., light, medium, heavy). The geometry is to distinguish between real (triangle) and projected aircraft positions. If there are multiple parallel runway centerlines, these will be reflected in the number of CSRs. In MINIMA, all arriving aircraft will be projected on the CSR. It will not show aircraft only on the Centerline. All objects move from the right to the left of the display depending on their speed. The leftmost number shows the distance to threshold. The CSR is displayed below the radar situation on the same display.

\begin{tabular}{|c|c|c|c|c|c|c|}
\hline \multicolumn{2}{|c|}{$\begin{array}{ll}\text { AIZ441 } & \text { DLH254 }\end{array}$} & \multicolumn{4}{|c|}{ DLH1MF } & \multirow{2}{*}{$\begin{array}{c}\text { BAW956m } \\
\cup\end{array}$} \\
\hline 2.45 & $\nabla>2.45<\nabla$ & $>8.16<$ & $\nabla$ & & $>8.75<$ & \\
\hline SAA264 & & LH7HF & & DLH500 & & DLH5E \\
\hline $1.40 \nabla$ & $\langle 7.54\rangle$ & 口 & $\langle 8.01\rangle$ & $\cup$ & $>3.61<$ & $\nabla$ \\
\hline
\end{tabular}

Fig. 3. Centerline Separation Range

\section{- Sequence optimization:}

The AMAN calculates an optimized sequence for the arriving aircraft. The trajectories are generated in such a way that the aircraft arrive at the merging point according to the planned sequence. The AMAN adds aircraft entering the planning horizon of the AMAN to the list. However, except for adding new aircraft, the list will not be updated automatically. If ATCos would like to update the list, they have to modify the sequence manually (e.g., using drag and drop on a sequence display). Having the possibility to modify the sequence would allow offering a new kind of service to airlines. Airlines have additional restrictions that are not met today. Considering that increasing automation reduces the workload of controllers and that safety is kept on the same level, the services provided by ATCos could be increased. It could be possible for airlines to change sequences of their arriving aircraft if it only affects their own aircraft. This can only happen if the aircraft of one airline are in sequence and are controlled by the same ATCo. This additional task is triggered by airlines that already know the sequence of their arriving aircraft and would like to change. This could be simulated with an additional tool that provides the special demands issued by airlines and pilots.

- Advisories:

The trajectories generated by the Arrival Manager will have phases of climb/descent in altitude, increase/decrease in speed. In the MINIMA application 
example, the aircraft will enter new phases included in the agreed trajectory automatically. If the aircraft were guided conventionally, the ATCo would have to give a clearance if an aircraft needs to enter a new phase of climb/descent or change its speed. It is possible to extract these necessary controller commands from the trajectory. This functionality was developed to support the controller by showing these commands as "Advisories". The advisory for a controller can include aircraft callsign, command type, command value, and a countdown, when this command should be executed by the pilot. An example could be "AFR376 DESCEND Alt $400015 \mathrm{~s}$ " (see also Fig. 4). These advisories can either be displayed in a stack or directly at the radar label. As the aircraft follow their trajectories automatically and ATCos do not have to give clearance in the MINIMA use case, they could be unaware of the changes of aircraft behaviour. For example, an aircraft can start to descend without the ATCo noticing. In MINIMA, the "Advisories" could help to increase the ATCos awareness regarding the behaviour of the aircraft and changes in the flight profile, e.g., by showing these Advisories or by requesting the ATCo to confirm these Advisories. In case Advisories are not confirmed, the related changes of the flight profile are not executed by the aircraft. These aircraft are no longer guided automatically, but have to be guided manually by the ATCo.

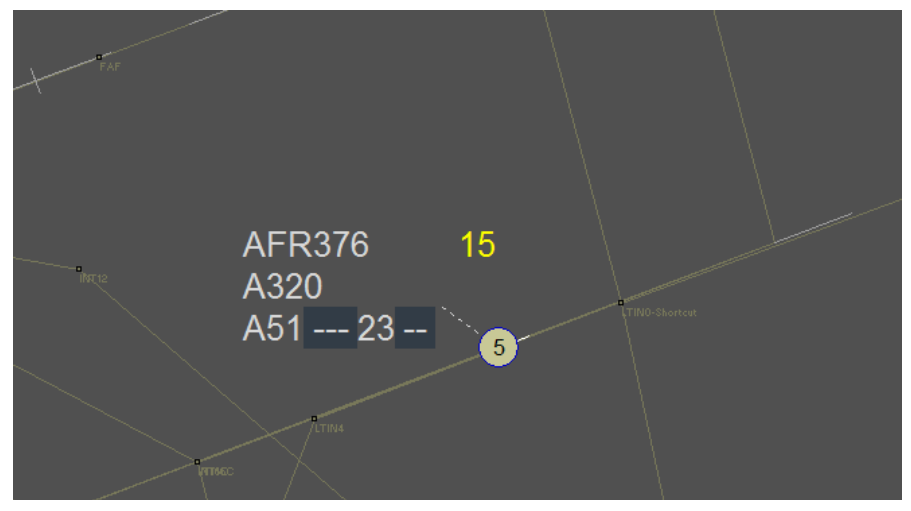

Fig. 4. Advisory countdown (yellow) shown at the controller radar display

- Provision of additional information to increase service.

This idea is also considering that the safety is kept on the same level trough automation and that the ATCo has more time to provide better service. The ATCo should provide the aircraft with additional information that are not provided today and that could be of relevance for the pilots. The task is triggered by the pilots that request the information. The ATCo then can decide if his workload allows him to provide the requested information. Examples for requests are: predicted minimum separation, additional weather information, or the parking position.

- Adaptation of Sector Size:

A common method to balance the workload of ATCos today is splitting or merging their airspace sectors. If high amounts of traffic are expected, the sector is split so 
that the traffic can be handled by two controller positions. The same could be applied in MINIMA. In principle, two types of adaptation of the sector size are possible. At first, the ATCo in the MINIMA use case can either be responsible for one or for both runways of the simulated airport. Further, it is possible to hand over aircraft from adjacent sectors earlier. The ATCo would be responsible longer for each individual aircraft and consequently would have to handle more aircraft at a time. In the next steps of this project, we will implement and test the solution proposed.

\section{Conclusion}

An increase of automation in air traffic control can have negative effects on the air traffic controller's performance. The effects are known as out-of-the-loop phenomenon. The MINIMA Project will develop a vigilance and attention controller to mitigate these effects. A highly automated arrival management task will be used as a case study. Psychophysiological measurements like EEG will be used to identify the state of the Air Traffic Controller and combined with adaptive task activation. This will allow for activating tasks based on the Air Traffic Controllers state to keep their performance on a high level and to ensure safe operations.

Acknowledgement. This project has received funding from the SESAR Joint Undertaking under grant agreement No. 699282 under European Union's Horizon 2020 research and innovation program. This paper reflects only the authors' view and the $\mathrm{JU}$ is not responsible for any use that may be made of the information it contains.

\section{$6 \quad$ References}

1. Birkmeier, B., Korn, B. \& Kügler, D. (2010). Sectorless ATM and Advanced SESAR Concepts: Complement not Contradiction. In: Digital Avionics Systems (DASC), 2010 IEEE/AIAA 29th, IEEE

2. Neal, A., Flach, J., Mooij, M., Lehmann, S., Stankovic, S. \& Hasenbosch, S. (2011). Envisaging the Future Air Traffic Management System. In: The International Journal of Aviation Psychology 21, 1, S. 16-34.

3. SESAR, "The roadmap for delivering high performing aviation for Europe European ATM Master Plan," Brussels, 2015.

4. M.R. Endsley and E.O. Kiris, "The Out-of-the-Loop performance problem and level of control in automation," in Human Factors: The Journal of the Human Factors and Ergonomics Society, 1995, pp. 381-394.

5. Endsley, M.R., \& Kiris, E. O. (1995). The out-of-the-loop performance problem and level of control in automation. Human Factors: The Journal of the Human Factors and Ergonomics Society 37(2), 381-394.

6. Kaber, D. B., \& Endsley, M. R. (1997). Out-of-the-loop performance problems and the use of intermediate levels of automation for improved control system functioning and safety. Process Safety Progress, 16(3), 126-131. 
7. Merat, N., \& Jamson, A.H. (2009). How do drivers behave in a highly automated car. In Proceedings of the 5th International Driving Symposium on Human Factors in Driver Assessment, Training and Vehicle Design, pp. 514-521.

8. Norman, S. D., \& Orlady, H. W. (1989). Flight deck automation: Promises and realities.

9. Rasmussen, J., \& Rouse, W.B. (1981). Human Detection and Diagnosis of System Failures.

10. Wiener, E.L., \& Curry, R.E. (1980). Flight-deck automation: Promises and problems. Ergonomics, 23(10), 995-1011.

11. Endsley, M. R. (1988). Design and evaluation for situation awareness enhancement. In Proceedings of the Human Factors and Ergonomics Society Annual Meeting (Vol. 32, No. 2, pp. 97-101).

12. Wickens, C. D. (1992). "Engineering Psychology and Human Performance," 2nd ed., Harper Collins, New York.

13. Endsley, M. R. \& Rodgers, M.D. (1998). Distribution of attention, situation awareness and workload in a passive air traffic control task: Implications for operational errors and automation. Air Traffic Control Quarterly, 6 (1) $21-44$.

14. Galster, S. M., Duley, J. A., Masalonis, A. J., \& Parasuraman, R. (2001). Air traffic controller performance and workload under mature free flight: Conflict detection and resolution of aircraft self-separation. The International Journal of Aviation Psychology, 11(1), 71-93.

15. Wickens, C. D., \& Kessel, C. (1979). The effects of participatory mode and task workload on the detection of dynamic system failures. IEEE Transactions on Systems, Man, and Cybernetics, 9(1), 24-34.

16. Billings, C. E. (1997). Aviation automation: The search for a human-centered approach.

17. Funk, K., Lyall, B., Wilson, J., Vint, R., Miemcyzyk, M., and Suroteguh, C. (1999). Flight deck automation issues. International Journal of Aviation Psychology, 9, 125-138.

18. Sarter, N. B., \& Woods, D. D. (1995). How in the world did we ever get into that mode? Mode error and awareness in supervisory control. Human Factors: The Journal of the Human Factors and Ergonomics Society, 37(1), 5-19.

19. Sarter, N. B., Woods, D. D., \& Billings, C. E. (1997). Automation surprises. Handbook of human factors and ergonomics, 2, 1926-1943.

20. Degani, A., \& Heymann, M. (2000). Some formal aspects of human automation interaction. NASA Technical Memorandum number 209600. Moffett Field, CA: NASA Ames Research Center.

21. Palmer, E. (1999). Murphi busts an altitude: A murphi analysis of an automation surprise. In Digital Avionics Systems Conference, 1999. Proceedings. 18th (Vol. 1, pp. 4-B). IEEE.

22. Van Charante, E. M., Cook, R. I., Woods, D. D., Yue, L., \& Howie, M. B. (1992). Human-computer interaction in context: Physician interaction with automated intravenous controllers in the heart room. Analysis, design, and evaluation of man-machine systems, 263-274. 
23. Davies, D. R., \& Parasuraman, R. (1982). The psychology of vigilance. Academic Pr.

24. Mosier, K. L., Skitka, L. J., \& Korte, K. J. (1994). Cognitive and social psychological issues in flight crew/automation interaction. Human performance in automated systems: Current research and trends, 191-197.

25. Gerbert, K., \& Kemmler, R. (1986). The causes of causes: determinants and background variables of human factor incidents and accidents. Ergonomics, 29(11), 1439-1453.

26. Matthews, G., \& Davies, D. R. (2001). Individual differences in energetic arousal and sustained attention: A dual-task study. Personality and individual Differences, 31(4), 575-589

27. Mackworth, N.H. (1948). The breakdown of vigilance during prolonged visual search. Quarterly Journal of Experimental Psychology, 1(1), 6-21.

28. Parasuraman, R. (1979). Memory load and event rate control sensitivity decrements in sustained attention. Science, 205(4409), 924-927.

29. Warm, J.S. (1984). An introduction to vigilance. Sustained attention in human performance, 1-14.

30. O'Hanlon, J. F. (1981). Boredom: Practical consequences and a theory. Acta psychologica, 49(1), 53-82.

31. Strauch, B. (2002). Investigating human error: Incidents, accidents, and complex systems. Burlington, VT:Ashgate.

32. Wiener, E.L. (1988). Cockpit automation. In E.L. Wiener and D.C. Nagel (Eds.), Human factors in aviation (pp.433-461). San Diego: Academic.

33. Parasuraman, R., Molloy, R., \& Singh, I. L. (1993). Performance consequences of automation induced 'complacency'. The International Journal of Aviation Psychology, 3(1), 1-23.

34. Rovira, E., McGarry, K., \& Parasuraman, R. (2007). Effects of imperfect automation on decision making in a simulated command and control task. Human Factors: The Journal of the Human Factors and Ergonomics Society, 49(1), 76-87.

35. Parasuraman, R., \& Riley, V. (1997). Humans and automation: Use, misuse, disuse, abuse. Human Factors: The Journal of the Human Factors and Ergonomics Society, 39(2), 230-253.

36. Moray, N., \& Inagaki, T. (2000). Attention and complacency. Theoretical Issues in Ergonomics Science, 1(4), 354-365.

37. Sheridan, T.B., Parasuraman, R., (2006). Human-automation interaction. In: Nickerson, R.S. (Ed.), Reviews of Human Factors and Ergonomics, vol. 1. Human Factors and Ergonomics Society, Santa Monica, CA, pp. 89-129.

38. Endsley, M. R. (1996). Automation and situation awareness. Automation and human performance: Theory and applications, 163-181.

39. Bahner, J. E., Hüper, A., \& Manzey, D. (2008). Misuse of automated decision aids: Complacency, automation bias and the impact of training experience. In International Journal of Human-Computer Studies, 66 (9), 688-699.

40. Bruder, C., Eißfeldt, H., Maschke, P., \& Hasse, C. (2014). A Model for Future Aviation. Aviation Psychology and Applied Human Factors, 4(1), 13-22. 
41. Parasuraman, R., Sheridan, T.B, \& Wickens, C.D (2000). A model for types and levels of human interaction with automation. In IEEE Trans. Syst., Man, Cybern. A 30 (3), pp. 286-297.

42. Dekker, S. W., \& Woods, D. D. (2002). MABA-MABA or abracadabra? Progress on human-automation co-ordination. Cognition, Technology \& Work, 4(4), 240244.

43. Bainbridge, L. (1983). Ironies of automation. In Automatica 19 (6), pp. 775-779.

44. Rieth, C. A., Cook, M. B., \& Ngo, M. K. (2015, August). Displays for effective human-agent teaming: evaluating attention management with computational models. In International Conference on Virtual, Augmented and Mixed Reality (pp. 216-227). Springer International Publishing.

45. Klein, G., Woods, D. D., Bradshaw, J. M., Hoffman, R. R., \& Feltovich, P. J. (2004). Ten challenges for making automation a" team player" in joint humanagent activity. IEEE Intelligent Systems, 19(6), 91-95.

46. Scerbo, M.W. (2006). Adaptive automation. In Waldemar Karwowski (Ed.): International encyclopedia of ergonomics and human factors. 2nd ed. Boca Raton, FL: CRC/Taylor \& Francis, pp. 1893-1896.

47. Parasuraman, R., Bahri, T., Deaton, J. E., Morrison, J. G., \& Barnes, M. (1992). Theory and design of adaptive automation in aviation systems. Naval Air Warfare Center. Warminster, PA, USA (Rep., NAWCADWAR-92 033-60).

48. St. John, M., Kobus, D.A., Morrison, J.G., and Schmorrow, D. (2004). "Overview of the DARPA augmented cognition technical integration experiment," in International Journal of Human-Computer Interaction, 17(2), pp. 131-149.

49. Wilson, G. F., \& Russell, C. A. (2003). Real-time assessment of mental workload using psychophysiological measures and artificial neural networks. Human Factors: The Journal of the Human Factors and Ergonomics Society, 45(4), 635-644.

50. Molloy, R., \& Parasuraman, R. (1996). Monitoring an automated system for a single failure: Vigilance and task complexity effects. Human Factors: The Journal of the Human Factors and Ergonomics Society, 38(2), 311-322.

51. Hawley, J. K. (2006). Patriot fratricides: The human dimension lessons of Operation Iraqi Freedom. FA JOURNAL, 11(1), 18.

52. Eggemeier, F. T. (1988). Properties of workload assessment techniques. Advances in psychology, 52, 41-62.

53. Kramer, A.F. (1991). Physiological metrics of mental workload: A review of recent progress. Multiple-task performance, 279-328.

54. Wilson, G. F., \& Russell, C. A. (2003). Real-time assessment of mental workload using psychophysiological measures and artificial neural networks. Human Factors: The Journal of the Human Factors and Ergonomics Society, 45(4), 635-644.

55. Makeig, S., Gramann, K., Jung, T. P., Sejnowski, T. J., \& Poizner, H. (2009). Linking brain, mind and behavior. International Journal of Psychophysiology, 73(2), 95-100.

56. Lin, C. T., Chuang, C. H., Huang, C. S., Tsai, S. F., Lu, S. W., Chen, Y. H., \& Ko, L. W. (2014). Wireless and wearable EEG system for evaluating driver vigilance. IEEE Transactions on Biomedical circuits and systems, 8(2), 165-176. 
57. Zhang, Z., Luo, D., Rasim, Y., Li, Y., Meng, G., Xu, J., \& Wang, C. (2016). A vehicle active safety model: vehicle speed control based on driver vigilance detection using wearable EEG and sparse representation. Sensors, 16(2), 242.

58. Oken, B. S., Salinsky, M. C., \& Elsas, S. M. (2006). Vigilance, alertness, or sustained attention: physiological basis and measurement. Clinical Neurophysiology, 117(9), 1885-1901.

59. Foxe, J. J., \& Snyder, A. C. (2011). The role of alpha-band brain oscillations as a sensory suppression mechanism during selective attention. Frontiers in psychology, 2, 154.

60. Frey, J. N., Ruhnau, P., \& Weisz, N. (2015). Not so different after all: The same oscillatory processes support different types of attention. Brain research, 1626, 183-197.

61. Jensen, O., Kaiser, J., \& Lachaux, J.P. (2007). Human gamma- frequency oscillations associated with attention and memory. Trends Neurosci. 30, 317-324.

62. Steriade, M., Gloor, P., Llinas, R.R., Da Silva, F. L., Mesulam, M.M. (1990). Basic mechanisms of cerebral rhythmic activities. In: Electroencephalogr. Clin. Neurophysiol., vol. 76, no. 6, pp. 481-508.

63. Makeig, S., Elliott, F., \& Postal, M. (1993). First demonstration of an alertness monitoring. management system," Tech. Rep, 93-36.

64. Ji, H., Li, J., Cao, L., \& Wang, D. (2011). A EEG-Based brain computer interface system towards applicable vigilance monitoring. In Foundations of Intelligent Systems (pp. 743-749). Springer Berlin Heidelberg.

65. Delorme, A., and Makeig, S. (2004). EEGLAB: an open source toolbox for analysis of single-trial EEG dynamics including independent component analysis. J. Neurosci. Methods 134, 9-21.

66. Klimesch, W. (1999). EEG alpha and theta oscillations reflect cognitive and memory performance: a review and analysis. Brain research reviews, 29(2), 169195, 1999.

67. Aricò, P., Borghini, G., Di Flumeri, G., Colosimo, A., Pozzi, S., Babiloni, F. (2016). A passive brain-computer interface application for the mental workload assessment on professional ATCOs during realistic air traffic control tasks. Progress in Brain Research, 2016.

68. Simons, D. J., Levin, D. T.: Change blindness. In: Trends Cogn. Sci., vol. 1, no. 7, pp. 261-267, Oct. 1997.

69. Stern, J. A., Boyer, D. J., Schroeder, D. J., Touchstone, R. M., Stoliarov, N. (1996). Blinks, Saccades; and Fixation Pauses During Vigilance Task Performance: 2: Gender and Time of Day. Mar. 1996.

70. Schaper, M., Temme, M.M., Gluchshenko, O., Christoffels, L., Pick, A. (2012). Coupling of ATM Planning Systems with Different Time Horizons / Air Transport and Operations Symposium 2012. Delft.

71. HungaroControl (2013). HungaroControl's MergeStrip process / HungaroControl. Budapest, 2013. 